\title{
Criminologie
}

\section{La perception du poker selon les joueurs adeptes : un jeu qui les distingue}

\section{Magali Dufour, Séverine Petit et Natacha Brunelle}

Volume 45, numéro 2, automne 2012

Crime et jeux de hasard

URI : https://id.erudit.org/iderudit/1013718ar

DOI : https://doi.org/10.7202/1013718ar

Aller au sommaire du numéro

\section{Éditeur(s)}

Les Presses de l’Université de Montréal

ISSN

0316-0041 (imprimé)

1492-1367 (numérique)

Découvrir la revue

Citer cet article

Dufour, M., Petit, S. \& Brunelle, N. (2012). La perception du poker selon les joueurs adeptes : un jeu qui les distingue. Criminologie, 45(2), 7-25.

https://doi.org/10.7202/1013718ar
Résumé de l'article

Présentement, le jeu de hasard et d'argent (JHA) gagnant le plus de popularité est le poker. Malgré les nombreux adeptes, peu de données scientifiques sur les habitudes de jeu des joueurs de poker et sur leur perception de ce loisir sont disponibles. Afin de décrire la perception qu'ont les joueurs de poker de leur activité, des entretiens qualitatifs ont été réalisés auprès de 20 d'entre eux. L'analyse des verbatims a permis de faire ressortir différents thèmes quant à leur vision des autres JHA, leur vision du poker, les différences entre les modalités du poker, le rôle du poker dans leur vie et les qualités nécessaires pour être un bon joueur de poker. À la lumière des expériences rapportées par les interviewés, trois grands constats se dégagent : 1 ) le poker est un jeu qui se distingue des autres JHA par plusieurs aspects ; 2) l'aspect légal ou illégal de l'activité ne semble pas faire partie des préoccupations des joueurs ; 3) la vision des joueurs de poker de leur activité est distincte de celle de leurs proches. Malgré les limites de cette étude, le discours des participants souligne bien les spécificités du monde du poker. 


\title{
La perception du poker selon les joueurs adeptes: un jeu qui les distingue
}

\author{
Magali Dufour ${ }^{1}$ \\ Professeure agrégée \\ Faculté de médecine et de sciences de la santé, Université de Sherbrooke \\ Magali.Dufour@usherbrooke.ca \\ Séverine Petit \\ Faculté de l'éducation permanente (criminologie), Université de Montréal \\ petit.sevrine@umontreal.ca \\ Natacha Brunelle \\ Professeure titulaire \\ Département de psychoéducation, Université du Québec à Trois-Rivières \\ Natacha.Brunelle@uqtr.ca
}

RÉSUMÉ . Présentement, le jeu de hasard et d'argent (JHA) gagnant le plus de popularité est le poker. Malgré les nombreux adeptes, peu de données scientifiques sur les habitudes de jeu des joueurs de poker et sur leur perception de ce loisir sont disponibles. Afin de décrire la perception qu'ont les joueurs de poker de leur activité, des entretiens qualitatifs ont été réalisés auprès de 20 d'entre eux. L'analyse des verbatims a permis de faire ressortir différents thèmes quant à leur vision des autres JHA, leur vision du poker, les différences entre les modalités du poker, le rôle du poker dans leur vie et les qualités nécessaires pour être un bon joueur de poker. À la lumière des expériences rapportées par les interviewés, trois grands constats se dégagent: 1) le poker est un jeu qui se distingue des autres JHA par plusieurs aspects; 2) l'aspect légal ou illégal de l'activité ne semble pas faire partie des préoccupations des joueurs; 3) la vision des joueurs de poker de leur activité est distincte de celle de leurs proches. Malgré les limites de cette étude, le discours des participants souligne bien les spécificités du monde du poker.

MотS-CLÉs - Poker, jeux de hasard et d'argent, qualitatif, gambling, Internet.

1. Toute correspondance concernant cet article doit être adressée à madame Magalie Dufour.

Criminologie, vol. 45, n 2 (2012) 


\section{Introduction}

Selon le Code criminel canadien, le jeu en ligne serait illégal. Pourtant, une rapide expansion de l'offre des jeux de hasard et d'argent (JHA) sur Internet s'est opérée dans les dix dernières années. De fait, en novembre 2011 le site www.online.casinocity.com répertoriait 2632 casinos en ligne, dont 565 avaient une version française et acceptaient les joueurs canadiens. En 2011, la société d'État Loto-Québec lançait son propre site de JHA en ligne, Espacejeux, afin que les Québécois puissent «jouer sur un site légal» (Loto-Québec, 2011). De l'ensemble des jeux de hasard et d'argent sur Internet, celui gagnant le plus en popularité est sans nul doute le poker, particulièrement dans sa variante connue sous le nom de Texas Hold'em (Wiebe, 2006; Chevalier et Pastinelli, 2008; Shead et al., 2008; Wood et Griffiths, 2008). En fait, le poker, jeu complexe aux mille facettes puisqu'il implique à la fois des mathématiques, de la psychologie et du hasard (Siler, 2010), est omniprésent dans la société, tant à la télévision, dans les bars, les casinos que sur Internet. En 2009, l'enquête populationnelle québécoise indiquait que 4,7\% des adultes rapportaient avoir joué au poker dans la dernière année (Kairouz et Nadeau, 2010). Le nombre d'adeptes est encore plus important en Ontario puisque $21 \%$ des répondants de 18 ans et plus jouaient au poker pour de l'argent (Responsible Gambling Council, 2006).

L'engouement mondial observé pour le poker, parfois nommé par certains chercheurs "pokermania» (Chevalier et Pastinelli, 2008; Mitrovic et Brown, 2009), résulte notamment de l'augmentation fulgurante de la disponibilité de ce jeu et de sa grande couverture médiatique (Griffiths et al., 2006; Wood et al., 2007). De fait, les médias québécois et internationaux mettent régulièrement l'accent sur la victoire des gagnants des tournois de poker et de leur parcours vers cette réussite. Ainsi, une vague de popularité a suivi la victoire de Chris Moneymaker qui a gagné en 2003 son droit d'entrée aux World Series of Poker (WSOP) en pariant d'abord de petites mises sur Internet. Depuis, les médias québécois ont souligné les victoires de nombreux joueurs dont celle en 2006 de Phil «Le Dingue» Boucher et en 2010 de Jonathan Duhamel. Par ailleurs, les bourses substantielles dans les tournois et l'endossement de cette activité par des célébrités contribuent aussi à sa popularité (Shead et al., 2008; Liley et Rakow, 2009). Ainsi, de nombreux artistes tels Patrick Bruel, René Angelil et bien d'autres s'identifient dans les médias comme étant des joueurs de poker. Les médias et célébrités 
contribuent donc à augmenter la visibilité de cette activité tout en lui donnant un aspect glamour et convoité.

Malgré les nombreux adeptes, peu de données scientifiques sur les habitudes de jeu des joueurs de poker et sur leur perception de ce loisir sont disponibles (Shead et al., 2008; Bjerg, 2010; Hopley et Nicki, 2010). Jusqu'à présent, les premières recherches se sont surtout intéressées à décrire les joueurs de poker souvent comme des étudiants universitaires, ne jouant qu'à l'une ou l'autre des modalités (en salle ou en ligne) (Griffiths et Barnes, 2008; Shead et al., 2008; Griffiths et al., 2010). Les premiers résultats de recherche laissent penser que plusieurs joueurs de poker sont à risque de développer un problème de jeu pathologique, peut-être davantage les joueurs de poker en ligne (Wood et al., 2007; Shead et al., 2008; Jonsson, 2009; Hopley et al., 2011). Par exemple, l'étude de Wood et collaborateurs (2007), menée auprès de 422 étudiants universitaires britanniques qui se définissaient eux-mêmes comme étant des joueurs de poker Internet, rapporte des prévalences de $18 \%$ de joueurs pathologiques probables et de $30 \%$ de joueurs à risque (Wood et al., 2007). D'autres études réalisées auprès de joueurs de poker Internet rapportent aussi de fortes prévalences de jeu pathologique (entre $8 \%$ et $9 \%$ ) et de jeu problématique ( $12 \%$ à $38 \%$ ) en comparaison de celles rencontrées dans la population générale $(0,7 \%$ de joueurs pathologiques et $1,3 \%$ de joueurs problématiques) (Jonsson, 2009; Hopley et Nicki, 2010; Kairouz et Nadeau, 2010). Les problèmes de jeu chez les joueurs de poker semblent liés à un manque de discipline, à certaines de leurs croyances en ce qui a trait à la place du hasard, à des dépenses importantes, à une fréquence et un nombre d'heures consacrés au jeu plus élevés, au stress vécu et même au fait d'avoir un locus de contrôle interne (Bjerg, 2010; Griffiths et al., 2010; Hopley et al., 2011). En fait, chez les joueurs de poker, une grande variabilité étiologique des problèmes de jeu semble présente (Bjerg, 2010). Le poker et ses adeptes professionnels et amateurs posent donc différents défis tant en ce qui a trait à la compréhension de cette activité qu'aux trajectoires pouvant conduire au développement d'un problème de jeu (Bjerg, 2010; McCormack et Griffiths, 2012).

D'autres acteurs s'inquiètent aussi des méfaits possibles du poker. De fait, en 2010, l'Institut national de santé publique (INSPQ) a déposé un mémoire au ministre des Finances, s'inquiétant de l'impact de l'étatisation des JHA sur Internet au Québec. Dans ce mémoire, la vulnérabilité des joueurs de poker, et particulièrement de ceux jouant sur 
Internet, est mentionnée à plusieurs reprises. Ainsi, «les joueurs (de poker Internet) sont incités à une participation intensive au jeu qui implique des dépenses continues, sinon grandissantes, et les rend très vulnérables aux problèmes de jeu» (Papineau, 2010: 3). Du coup, ils recommandent un moratoire sur le développement des JHA, dont le poker, sur Internet (INSPQ, 2011).

Alors qu'il semble exister un écart important entre la vision médiatique et la vision de la santé publique, qu'en est-il de la perception des joueurs de poker eux-mêmes? Comment voient-ils cette activité ? Laspect légal de cette activité fait-il partie de leurs préoccupations? Sont-ils en mesure de nuancer et de voir les risques associés à cette activité? Outre les rares études qualitatives s'étant intéressées à des joueurs de poker professionnels (Radburn et Horsley, 2011; McCormack et Griffiths, 2012), peu d'études se sont attardées sur la vision et la compréhension de ce loisir du point de vue des joueurs considérés comme des acteurs sociaux (Debuyst, 1989). Or, le sens que les acteurs donnent à leur expérience (Savoie-Zajc, 1998) permet de comprendre les représentations et systèmes de valeurs propres à une sous-culture (Michelat, 1975). Selon la théorie de l'interactionnisme symbolique (Blumer, 1969), un objet peut représenter n'importe quoi, selon la signification qu'il a pour chacun. Aussi, dit-il, la définition que les autres ont d'un objet influence la signification de cet objet pour soi. Le monde est composé d'objets qui sont le produit de l'interaction symbolique. Cet article a donc pour objectif de décrire la perception qu'ont les joueurs de poker de leur activité.

\section{Démarche méthodologique}

Ces données sont issues d'une étude transversale mixte ${ }^{2}$ combinant une méthode quantitative et qualitative afin de documenter les habitudes de jeux de hasard et d'argent des joueurs de poker. Pour répondre aux objectifs de cet article, seules les données du volet qualitatif seront utilisées.

L'échantillon qualitatif a été créé à partir d'un échantillon de convenance de 200 joueurs de poker recrutés dans 16 régions du Québec (Dufour et al., 2009). Pour participer, les joueurs devaient être âgés d'au moins

2. Cette étude a été approuvée par le Comité d'éthique de la recherche avec des êtres humains: Lettres et sciences humaines de l'Université de Sherbrooke. 
18 ans, se définir comme des joueurs de poker et y jouer avec de l'argent réel. Afin de sélectionner ces participants, les candidats devaient: 1) jouer au poker sur Internet; 2) habiter à moins d'une heure d'un grand centre urbain ${ }^{3}$; 3) parler français. Afin d'obtenir la vision de l'ensemble des joueurs (du non-problématique au pathologique) et de ne pas surreprésenter un groupe, les joueurs sélectionnés pour ce volet qualitatif devaient reproduire la distribution obtenue par l'échantillon global à I'ICJE (Ferris et Wyne, 2001). Au total, 20 joueurs de poker ont participé à ces entrevues.

La quasi-totalité des interviewés sont des hommes (18 sur 20). Ils sont âgés entre 18 et 45 ans (moyenne: 28,8, écart-type de 8,5 ans). La moitié des joueurs sont en couple ou mariés. Le dernier niveau de scolarité complété est le secondaire pour le quart de l'échantillon, le collégial pour 8 personnes et 7 participants ont fait des études universitaires. Le revenu moyen des participants est de 29088,23 \$ (écarttype de $20437,58 \$$ ). Les trois quarts des joueurs se considéraient comme des joueurs intermédiaires, alors que 5 se percevaient comme étant des experts ou des professionnels. En ce qui a trait à la sévérité des problèmes de jeu tels qu'évalués par l'Indice canadien du jeu excessif (ICJE), 4 joueurs étaient considérés sans problème, 7 joueurs à faible risque, 7 joueurs à risque modéré et 2 joueurs pathologiques probables.

Des entretiens individuels semi-structurés ont été menés auprès de chacun de ces joueurs afin qu'ils partagent leur perspective de joueur de poker (Poupart, 1997) ${ }^{4}$. L'entrevue était divisée en deux parties. La première concernait les habitudes de jeux de hasard et d'argent dans leur ensemble. Ensuite, l'expérience spécifique du poker était explorée. L'intervieweur possédait une liste de thèmes couvrant les objectifs de l'étude et inspirée des écrits scientifiques sur le sujet ou de l'absence de documentation sur certains aspects (l'initiation au poker, les aspects positifs et négatifs du poker, le contexte de la pratique, les objectifs et motivations) à aborder sous forme de questions ouvertes si le joueur ne

3. Cette considération est d'ordre budgétaire. L'équipe de recherche se situant dans les régions de Montréal, Trois-Rivières et Québec, il était possible d'y conduire les entrevues pour mener à bien ce volet de la recherche dans les limites de la subvention.

4. La plupart des entretiens (18 sur 20) ont été menés par la deuxième auteure de cet article. Celle-ci poursuivait alors des études supérieures dans lesquelles cette méthode de collecte de données était privilégiée. Elle possédait également une formation d'orientation clinique (criminologie) l'outillant pour intervenir auprès de populations diversifiées. Elle avait également en sa possession les coordonnées d'intervenants spécialisés vers lesquels elle pouvait diriger le participant s'il en manifestait le besoin. 
le faisait pas spontanément. Rares sont les fois où ces thèmes ont été introduits par l'intervieweur, mis à part la perception des problèmes de jeu. Les entrevues ont été d'une durée moyenne de 90 minutes pour lesquelles le participant recevait un chèque-cadeau de $30 \$$. La saturation empirique a été atteinte après une quinzaine d'entretiens. Ceux-ci ont été enregistrés, retranscrits sous forme de verbatim en utilisant des noms fictifs, puis analysés pour comprendre l'expérience et les préoccupations des joueurs. L'analyse des entretiens semi-structurés a été réalisée selon la méthode d'analyse du contenu thématique (Miles et Huberman, 1994; Ghiglione et Matalon, 1998).

Chaque entretien a été enregistré pour être retranscrit dans le logiciel Nvivo 8.0. Une grille de codification a été développée, permettant ensuite l'analyse verticale des entretiens. Cette grille fut adaptée à partir de la grille d'entretien et du contenu émergeant des trois premiers entretiens afin de s'assurer de rester fidèle aux préoccupations et propos des participants. Sous la supervision des chercheures principales, les entrevues ont d'abord été codifiées dans une analyse verticale par une auxiliaire de recherche, puis par une seconde auxiliaire pour la moitié des entretiens. Cette façon de procéder a permis d'en arriver à un accord interjuge afin de peaufiner la grille de codification et de s'assurer d'une uniformisation de celle-ci entre les assistants de recherche qui codifiaient les verbatims. Par la suite, chaque thème a été repris pour procéder à une analyse transversale du matériel. C'est au cours de cette étape que les tendances qui seront ici présentées ont pu être mises en lumière.

\section{Résultats}

L'analyse des verbatims des joueurs de poker a permis de faire ressortir différents thèmes quant à leur vision des autres JHA, leur vision du poker, les différences entre les modalités du poker, le rôle du poker dans leur vie, les qualités nécessaires pour être un bon joueur de poker et la vision de leurs proches du poker.

Le poker vu par les joueurs

Le poker est différent des autres JHA

Malgré le fait que la majorité des participants a préalablement été initiée aux autres formes de JHA, les joueurs entretiennent un intérêt limité, voire pas d'intérêt du tout pour les autres JHA. Pour ces joueurs, tous 
les jeux (à l'exception du poker) sont perçus comme ennuyants. Les autres JHA ne sont que des activités de gambling et présenteraient plus de risques de pertes financières que de gains. Les joueurs reprochent aux autres types de jeu de ne présenter qu'une illusion de contrôle 5 . Contrairement au poker, il leur est impossible de s'améliorer. Les joueurs de poker ont même parfois l'impression d'être floués par ces autres jeux:

[...] y'a aucun talent. Je veux dire ça pourrait être un enfant de 2 ans qui pèse sur le piton et il pourrait gagner, donc... Je ne me sens pas gratifié de jouer à ce jeu-là, je trouve ça un peu abrutissant. Quand on connaît les statistiques [...] parce qu'on pourrait dire, entre guillemets, «tu te fais fourrer» quand tu vas jouer sur les machines à sous parce qu'elles sont programmées pour prendre un certain pourcentage de l'argent que tu mets dans ta machine. $\left(\operatorname{Hugo}^{6}\right)$

La grande part de hasard perçue dans les autres JHA repousse donc les joueurs qui ont tendance à se spécialiser dans le poker.

\section{Le poker, jeu de stratégies}

L'aspect stratégique du poker semble être à la fois l'élément principal définissant le poker et un puissant facteur de motivation pour les joueurs. En fait, les entrevues ont permis de constater que les joueurs parlent abondamment des stratégies et des habiletés nécessaires tout en étant très sensibles à la controverse hasard versus habileté.

[...] Tout le monde pense que c'est juste de la chance, que c'est du gambling. Mais je te l'ai dit, dans ma tête à moi, ce n'est pas que du gambling; c'est un jeu de stratégies. Ceux qui disent que c'est du gambling, ils disent ça parce qu'ils ne savent pas jouer ou parce qu'ils n'ont jamais joué. Mais je pense que n'importe quel joueur de poker va te le dire: non, il n'y a pas juste de la chance. (Thierry)

Alors que tous s'accordent pour dire que le hasard est présent, l'habileté et, par conséquent, les stratégies utilisées par le joueur sont déterminantes dans les résultats qu'il obtiendra. Si à court terme la chance influence le résultat de quelques mains, ce sont, selon ces joueurs, les aptitudes et stratégies qui décideront d'un vrai gagnant sur le long terme:

5. L'illusion de contrôle est une surestimation de la probabilité de gagner par rapport à ce que la probabilité objective garantit. La personne surestime ses chances de gagner. Elle pense donc être capable d'influencer l'issue du jeu par son habileté, son expérience ou encore ses observations (Ladouceur et al., 2000).

6. Tous les noms utilisés sont fictifs. 
[...] tous les joueurs compétents vont vous dire que c'est sûr qu'il y a une grosse partie de chance à court terme, à long terme c'est vraiment... les habiletés bon, qui priment, qui prévalent là. (Didier, p. 10)

Le poker, source de revenus

Largent est un concept central au poker et récurrent dans le discours des joueurs, souvent conceptualisé non pas comme une dépense, mais comme un investissement à faire croître. De fait, les entrevues ont permis de constater que la motivation pécuniaire est particulièrement présente lorsque le jeu est pratiqué sur Internet, qui est considéré comme étant plus rapide que le jeu sur table. Certains expliquent que sans l'éventualité d'un profit, ils ne perdraient pas leur temps à y jouer: «Je suis pas si sûr que je jouerais sur Internet si je ne faisais pas d'argent» (Benoît). Effectivement, environ la moitié des joueurs considère le poker sur Internet comme une source de revenus (principal ou d'appoint):

J'essaie aussi de voir ça comme une source de revenus supplémentaires. À chaque deux semaines, je vais essayer de commander mon soixante-quinze dollars. J'essaie d'équilibrer ça; à chaque fois que j'ai une paye à la job, je veux recevoir ce chèque-là en même temps. (Rémi)

Les différentes formes de poker: distinctions et similitudes

En début d'entretien, les joueurs tenaient fréquemment à bien «éduquer» l'intervieweur quant aux différentes formes de poker. Si presque tous pratiquent les deux modalités, ils distinguent le jeu sur table (ou en salle) de celui sur Internet. Sur Internet, les stratégies de jeu doivent être adaptées en fonction de l'absence physique des adversaires, éliminant ainsi la possibilité de lire le langage non verbal. Le joueur, s'il bénéficie du confort de la maison, doit également savoir apprécier la cadence accélérée des parties. Ce rythme plus rapide que celui en salle est vu par certains comme offrant davantage de possibilités de gains monétaires. Des interviewés soulignent également la possibilité de jouer à un faible coût.

Une seconde distinction dichotomique émerge du discours des joueurs: l'existence des tournois et des cash games ${ }^{7}$. Tous s'adonnent aux

7. Dans un tournoi, tous les joueurs donnent un même montant d'argent au départ et jouent jusqu'à ce qu'ils soient éliminés. En revanche, en cash game, un joueur peut s'asseoir 
deux formes, mais soulèvent des nuances qu'ils relient principalement au contrôle de l'argent injecté et retiré:

J'en ai fait quelques-unes, des cash games. Entre autres, la fois où j'avais bu. Et j'ai vraiment tout perdu l'argent que j'avais ce soir-là. Ça fait que ça ne m'intéresse pas les cash games. Je préfère faire des tournois, même sur Internet. Je préfère participer à des tournois, parce que je joue pour l'apprentissage. Et le montant que tu mets, t'en mettras pas plus. (Jean)

De plus, bien que les avis soient partagés, plusieurs considèrent que si les gains peuvent être accrus au poker pratiqué sous la forme de cash games, les pertes seraient également plus importantes.

Le poker: un loisir ou un travail?

Le poker est parfois vu comme un loisir, parfois comme une forme de travail. Dans tous les cas, le poker est perçu par les joueurs comme un événement important dans leur vie:

J'ai mon emploi, je fais mes affaires et j'ai toujours plein de projets. Mais le poker, c'est une révélation dans ma vie. Un jour, je vais faire un gain important au poker et je ne suis pas certaine, mais un jour je pourrais gagner ma vie avec ça. (Lilianne)

Le poker sur table, lorsque pratiqué entre amis, est particulièrement associé à la notion de loisir. Le poker y est alors comparé à tout autre divertissement dans lequel argent et temps sont investis pour obtenir du plaisir:

On peut accepter le fait que ça va coûter de l'argent pour jouer. Toutes sortes de divertissements coûtent quelque chose; si on va au cinéma, ça coûte tant d'argent, si on va voir une pièce de théâtre, ça coûte de l'argent... il y a toujours des frais associés [...]. Je veux dire: payer vingt dollars, je pense que personne va avoir de problème à faire ça pour une activité d'une soirée. (Luc)

Certains joueurs associent plutôt le poker, principalement lorsque pratiqué en ligne, à la notion de travail. Lorsque c'est le cas, même si la régularité de la pratique demeure, l'intérêt tend parfois à diminuer à cause de la discipline nécessaire et du stress généré:

à une partie à n'importe quel moment et la quitter quand bon lui semble. Il peut également injecter de l'argent dans la partie aussi souvent qu'il le souhaite. 
Quand c'est un jeu, c'est le fun. Quand c'est un travail, c'est plate! Tu viens que l'intérêt que tu portes au jeu comme tel... Avant, je jouais sur quatre tables à la fois. J'avais des situations qui étaient nouvelles pour moi et j'essayais de savoir c'était quoi le meilleur jeu à faire, je pouvais essayer d'analyser certaines décisions que j'avais prises. Maintenant je dois en jouer à peu près 12,16 tables à la fois. Et la majorité des décisions que je prends sont robotiques, c'est-à-dire que je ne les ai pas analysées ces mains-là, je le sais ce que je dois faire. Le fait que ce soit un emploi, je vois ça comme un travail à la chaîne dans le fond. Et ça, c'est un peu plate, parce que tu perds un peu le fun du jeu. Ça reste que je m'intéresse toujours au poker, que j'aime jouer, que je continue à apprendre tous les jours sur le poker quand même. C'est juste que l'intérêt est moins élevé que lorsque j'ai commencé, ou que lorsque je jouais juste pour le fun. (Guillaume)

\section{Pour devenir un bon joueur}

Le poker, qu'il soit vu comme un loisir ou comme un travail prend une place considérable dans la vie de certains participants qui souhaitent tous s'améliorer au fil de leur pratique. Désireux d'améliorer leurs stratégies de jeu, les participants soulignent les multiples outils (lectures, logiciels, participation à des blogues, coach, émission de télévision, etc.) utilisés pour devenir un meilleur joueur:

Donc, il faut que je m'améliore. Je lis des livres sur le poker. J'étudie mes jeux. C'est vraiment plus que simplement ouvrir, puis commencer à jouer. Il y a une part de jeu, mais il y a une part d'apprentissage aussi: j'étudie le poker. (Benoît)

Non seulement discipliné dans son apprentissage, selon les interviewés, le bon joueur est celui qui demeure attentif et concentré sur tout ce qui l'entoure, le poker étant un jeu d'interactions. Il doit aussi être en mesure d'estimer ses compétences et de connaître ses limites afin de s'en tenir à un niveau de jeu contrôlé. Par ailleurs, le bon joueur est également celui qui a développé une discipline dans la gestion de son argent. Ainsi, il saura évaluer le capital dont il dispose, jouera à des limites financières conséquentes et sera capable d'absorber certaines pertes. Enfin, il doit savoir garder son sang-froid dans toutes les situations et faire preuve de patience pendant les parties. Il ne peut se laisser submerger par le «thrill», le stress ou les déceptions.

Un bon joueur, c'est quelqu'un qui va savoir jouer, qui va savoir bien jouer, qui va savoir lire ses adversaires, qui va savoir quand mettre l'argent, quand 
pas mettre de l'argent, qui va pouvoir analyser tout le monde sur la table, prendre des notes sur tout le monde. Il va mémoriser à chaque main. (Olivier)

Cette discipline à tous les niveaux et de tous les instants s'avère la pierre angulaire de la réussite au poker.

\section{La façon dont les joueurs se sentent perçus}

Certains joueurs ont des proches et des conjoints qui les encouragent à jouer. Ces proches considèrent que c'est une façon de faire de l'argent et que le joueur est "prudent». Les joueurs apprécient cette confiance, particulièrement dans les moments de défaite:

Elle est super. Elle est positive là-dessus. Elle dit: «Non, non, c'est bon, joue. C'est bon, tu fais de l'argent!» Elle a vu toute l'évolution. Elle voit que je gagne tout le temps. Elle a confiance. Ça ne l'énerve pas. (Benoît)

Alors que certains joueurs se sentent soutenus par leur famille, plusieurs participants croient que leurs proches ne partagent pas leur propre vision du poker. Au contraire, ils ont l'impression que leurs proches entretiennent plusieurs préjugés face à l'activité présentée comme répréhensible et dangereuse :

Au début ils disaient: «Le jeu, c'est mal...» Le jeu, c'est pas mal. L'alcool, c'est pas mal. Donc, arrêtez! C'est pas parce que tu bois une bière entre amis que t'es un alcoolique. C'est le même principe. Il ne joue pas l'argent, il gagne l'argent. Il ne met pas son argent et croise ses doigts en espérant que les dés vont être favorables aujourd'hui. Ce n'est pas exactement comme ça que ça se passe. (Étienne)

Selon les interviewés, certains membres de leur entourage ne verraient donc que les aspects négatifs de ce jeu, occultant les autres facettes. Certains joueurs rapporteront même être stigmatisés, jugés ou perçus automatiquement comme des joueurs pathologiques parce qu'ils pratiquent cette activité. Ces jugements négatifs seront parfois difficiles à vivre pour les joueurs:

Mais tu sais, au début, c'était clair que je recevais des commentaires négatifs. Là je disais: «Je joue au poker!» On me répondait: «Ah! Mon Dieu! Là tu vas perdre tout ton argent, tu es une gambler... ça c'est certain que le poker...» Quand tu joues au poker, t'as l'air d'une personne qui est irresponsable, qui va tout perdre, que nécessairement elle va jouer et jouer et jouer et que faire ça. Mais bon, pas nécessairement là. (Roxanne) 
Les joueurs ont l'impression que les autres ne comprennent pas les spécificités du poker, particulièrement l'habileté nécessaire à ce jeu. Cette méconnaissance expliquerait, en partie du moins, leurs préjugés négatifs. Afin de modifier cette perception, certains joueurs tenteront d'expliquer le jeu en insistant sur la part de stratégie dans le jeu. À titre d'exemple, Christian explique la façon dont il approche le sujet avec son père:

Je lui en parle, mais au début chaque fois que je parlais de poker, il voulait toujours éviter le sujet. Tranquillement, je lui parle, je lui montre un peu comment ça fonctionne. Je lui montre qu'il y a des statistiques à travers ça, qu'il y a beaucoup de mathématiques aussi dans le poker. Puis de plus en plus, je vois tranquillement une ouverture. C'est ça, j'essaie d'en discuter, de lui en parler à chaque fois que je le vois. (Christian)

La vision négative du poker des proches serait aussi tributaire, selon certains interviewés, de la représentation que font les médias du poker et des joueurs de poker. Ainsi, les joueurs ont l'impression que les médias, en ne rapportant que les méfaits associés au poker, contribueraient aux préjugés de la population.

$\mathrm{Au}$ début, elle était réticente par rapport à ça. Mais c'est beaucoup de préjugés au poker. Le monde, les médias, tout ce qu'ils projettent de l'argent, du jeu, c'est que c'est un jeu de hasard. Oui, en tant que tel, il y a de la chance. Mais c'est quoi la chance? Là-dedans, c'est $10 \%$ de $90 \%$. Je veux dire, à long terme, le bon joueur va tout le temps sortir gagnant comparativement à celui qui est juste chanceux. Et c'est beaucoup de préjugés par rapport à ça. Les médias, ce qu'ils envoient c'est ça et le monde ils croient juste ça aujourd'hui. Et ils disent: «Ah! beaucoup de joueurs en meurent là, et c'est juste du compulsif.» Et c'est pas vrai. Et dès que tu commences à comprendre c'est quoi vraiment le jeu en tant que tel, la personne ne peut pas faire autrement que de comprendre pourquoi t'es là-dedans. (Olivier)

Enfin, les chercheurs aussi auraient de fausses représentations du monde du poker selon des répondants. Une fois de plus, certains joueurs ont l'impression d'une représentation erronée, basée sur des préjugés. Leur réponse à notre annonce pour le recrutement affichée sur un forum regroupant des joueurs en est un exemple.

Alors pourquoi est-ce que moi ou quiconque ici se porterait volontaire à cette étude quand le but n'est pas de valoriser le poker québécois en général, mais bien de l'associer et faire des corrélations avec le gambling en général. (sic) (décembre 2007) 
En bref, les joueurs de poker ont l'impression que leurs proches, les médias et les chercheurs ont souvent une vision alarmiste et déformée du poker.

\section{Discussion et conclusion}

À la lumière des expériences rapportées par les interviewés, il est possible de dégager trois grands constats. D'abord, le poker est un jeu qui se distingue des autres jeux de hasard et d'argent (JHA) et ce, sous plusieurs aspects. Dans un deuxième temps, l'aspect légal ou illégal de l'activité ne semble pas faire partie des préoccupations des joueurs. Enfin, la vision des joueurs de poker sur leur activité est distincte de celle de leurs proches et des acteurs en santé publique.

Tout d'abord, il semble clair, pour l'ensemble des joueurs, que le poker est un JHA spécifique et différent qui se distingue de plusieurs façons des autres formes de JHA. Les JHA ne peuvent donc pas, du point de vue des participants, et de certains chercheurs, être conceptualisés comme étant des activités homogènes présentant les mêmes facteurs de risque (Dickerson, 1993; Bjerg, 2010; Lloyd et al., 2010). En fait, des chercheurs vont même jusqu'à suggérer que des processus psychologiques différents et spécifiques seraient mis en œuvre lors du développement du jeu pathologique selon le type de jeu (Dickerson, 1993; Petry, 2005). Selon Bjerg (2010), les spécificités structurelles du poker auraient donc potentiellement des implications directes sur la façon dont les problèmes de jeu pourraient émerger chez les joueurs de poker. L'hétérogénéité observée et vécue par les participants remet donc en question la généralisation des résultats réalisés auprès des joueurs d'appareils de loterie vidéo.

Non seulement le poker est différent des autres JHA, mais ses modalités (en salle et en ligne) et ses différentes formes (tournoi vs cash game) influencent aussi les stratégies et facteurs de risque des joueurs de poker. De fait, les joueurs de poker font des distinctions importantes entre leur pratique sur Internet et celle sur table. Ainsi, le poker Internet est parfois comparé à un travail exigeant, sans plaisir, centré sur l'argent, où ils doivent jouer à plusieurs tables pour réussir. Quant à la forme du cash game, elle semble associée à une plus grande prise de risque chez les joueurs de poker. Ces distinctions dans le poker soulignent l'importance de bien préciser les modalités (Internet vs salle) et les formes (cash game vs tournoi) de poker lors des futures recherches. 
Malgré l'introduction du thème lors des entrevues, l'aspect légal ou illégal est complètement inexistant de leur vision de ce jeu et de leurs préoccupations. En fait, aucun de ces joueurs n'a soulevé la question de la légalité de cette activité, notamment dans sa modalité Internet. Pourtant, tous avaient joué dans la dernière année sur Internet. Or, selon Loto-Québec, les personnes jouant sur des sites Internet autres que celui d'Espacejeux joueraient sur des sites illégaux. En fait, «misez en toute légalité» est le premier argument de Loto-Québec pour attirer les joueurs sur son site Internet (Loto-Québec, 2011). La vision de la société d'État sur les joueurs de poker jouant sur Internet laisse donc penser qu'ils posent un geste illégal, vision que ne semblent pas partager les joueurs de poker.

L'aspect le plus important pour les joueurs de poker semble être la composante habileté présente dans cette activité. De fait, les joueurs furent éloquents sur cette composante et son importance dans la compréhension du poker. Sans la composante habileté et les stratégies qui en découlent, le poker ne serait qu'un jeu parmi d'autres. Alors que les participants reconnaissent la place du hasard dans le poker, tous s'entendent pour dire que la stratégie est la pierre angulaire de la réussite dans cette activité. Cette vision influence à la fois les motivations et le temps investi dans l'activité. Par ailleurs, la présence d'habiletés et de progression possible s'avère aussi une des fondations de «l'impression de contrôle» (parfois nommée illusion de contrôle dans les autres JHA). De fait, les joueurs tentent par différentes stratégies (lecture, études, pratique, etc.) d'améliorer leur jeu en diminuant la place accordée au hasard. Le risque dans ce jeu unique où l'habileté est véritablement présente est que le joueur oublie la partie hasard et surestime le contrôle qu'il peut exercer. Or, de nombreuses études ont associé l'illusion de contrôle au développement d'un problème de jeu pathologique (Joukhador et al., 2003; Blasczynsky et Nower, 2007; Mattson et al., 2008). Compte tenu de la sensibilité des joueurs de poker sur la question de l'habileté, il est donc très important, pour l'ensemble des intervenants, d'être en mesure de reconnaitre à la fois la composante hasard et la place de l'habileté. Ce n'est que de cette façon que le joueur de poker aura l'impression que son activité est reconnue et comprise par son interlocuteur. En accordant de l'importance au regard que pose le joueur de poker sur cette activité, il est possible de suggérer des pistes d'intervention adaptées à sa réalité et aussi de nuancer le discours médiatique sur le sujet. 
Finalement, il existe une grande différence entre la vision qu'ont les joueurs de poker de leur activité de celle "des autres», à savoir leurs proches, les médias et les chercheurs. De fait, les joueurs voient davantage les aspects positifs au poker et la possibilité de gains financiers. Au contraire, leurs proches et les chercheurs, en plus de ne pas comprendre l'essence même de ce jeu, mettraient davantage l'accent sur les méfaits et les risques associés au poker selon eux. Quant aux médias, alors que les gagnants dans les tournois de poker reçoivent une couverture médiatique importante, certains joueurs ont l'impression que les médias font davantage ressortir le spectre du jeu pathologique. Que ce soit le regard des chercheurs, de leurs proches ou des médias, les joueurs de poker ont souvent l'impression que le jugement des autres est rempli de préjugés négatifs à l'encontre du poker. Puisque leur activité est incomprise, ils se doivent d'éduquer et de convaincre les nonjoueurs de la légitimité de leur activité et surtout de ses avantages. C'est d'ailleurs la raison principale pour laquelle la majorité des participants a accepté de rencontrer nos intervieweurs. Par ailleurs, cet écart dans les visions contribuerait à créer une stigmatisation ou un sentiment d'être jugés chez les joueurs de poker participants. Ce stigma pourrait, à long terme, créer des obstacles à l'entrée en traitement puisqu'un joueur de poker compulsif aura de la difficulté à dévoiler l'ampleur de ses pertes et de sa souffrance, ayant l'impression qu'il sera jugé. C'est là un bel exemple de la portée de l'interactionnisme symbolique (Blumer, 1969) selon lequel la signification qu'accordent les acteurs sociaux à un objet - ici le poker - est influencée par le regard des autres et entraîne des réactions et comportements. Il est possible alors, par exemple, de mieux comprendre ce qui peut nuire ou faciliter une demande d'aide ou une réceptivité à des interventions préventives ou curatives en cas de besoin.

Les résultats de cette recherche, bien que permettant d'avoir accès à la vision des joueurs de poker, doivent toutefois être interprétés dans les limites de cette étude. Le recrutement des joueurs de poker, particulièrement les joueurs Internet, fut ardu en raison d'une attitude méfiante face à la recherche sur le poker. En effet, ces joueurs craignent que les résultats de l'étude puissent nuire à l'image du poker, une activité qui les passionne. À ce titre, comme c'est le cas pour toute étude sur les JHA ou autres activités marginales, cette attitude a pu avoir un effet sur la validité des résultats. Il n'est pas exclu que la désirabilité sociale conduise des participants à minimiser les conséquences de leurs 
habitudes de poker dans les différentes sphères de leur vie. Il faut également prendre en compte que l'étude est basée sur un échantillon de convenance. Les joueurs rencontrés sont uniquement ceux ayant répondu à l'appel et nous ignorons le taux de réponse. Il est donc possible que nous n'ayons pas eu accès à tous les types de joueurs de poker.

En conclusion, le poker est une activité différente qui nécessite des études spécifiques auprès de ses adeptes. Les nombreuses spécificités du poker qui sont importantes aux yeux des joueurs soulignent la nécessité de s'attarder à l'ensemble des activités de poker et non pas seulement considérer cette activité comme un tout. De futures études devront donc prendre en compte tant les modalités (Internet ou en salle) que les formes de poker (cash games ou tournois). Ce n'est que de cette façon que l'on pourra avoir accès au monde complexe qu'est le poker.

\section{Références}

Bjerg, O. (2010). Problem gambling in poker: Money, rationality and control in a skill-based social game. International Gambling Studies, 10 (3), 239-254.

Blaszczynski, A., \& Nower, L. (2002). A pathways model of pathological and problem gambling. Addictions, 97 (5), 487-499.

Blumer, H. (1969). Symbolic Interactionism: Perspective and Method. New Jersey: Prentice-Hall.

Chevalier, S. \& Pastinelli, M. (2008, mai). L'offre de jeu de poker sous toutes ses coutures ou la sociologie du poker. Communication présentée au $76^{\mathrm{e}}$ congrès de l'ACFAS, Québec, Québec.

Debuyst, C. (1989). Acteur social et délinquance. Bruxelles: Pierre Mardaga.

Dickerson (1993). Internal and external determinants of persistent gambling: Problems in generalising from one form of gambling to another. Journal of Gambling Studies, 9 (3), 225-245.

Dufour, M., Brunelle, N., Richer, I. \& Petit, S. (2009). Le rôle du poker en ligne dans les trajectoires de jeu de hasard et d'argent. Rapport de recherche remis au FQRSC.

Ferris, J., \& Wynne, H. (2001). L'indice canadien de jeu excessif (Rapport final). Consulté le 15 mai 2007, http://www.ccsa.ca/CCSA/FR/Topics/Substances_Addictions/Gambling.htm.

Griffiths, M., \& Barnes, A. (2008). Internet gambling: An online empirical study among student gamblers. International Journal of mental bealth addiction, 6, 194-204.

Griffiths, M., Parke, A., Wood, R., \& Parke, J. (2006). Internet gambling: An overview of psychosocial impacts. UNLV Gaming Research and Review Journal, $10(1), 27-39$ 
Griffiths, M., Parke, A., Wood, R., \& Rigbye, J. (2010). Online poker gambling in university students: Further findings from an online survey. International Journal of Mental Health and Addiction, 8 (1), 82-89.

Hopley, A. B., Dempsey, K., \& Nicki, R. (2011). Texas hold'em online poker: A further examination. International Journal of Mental Health and Addiction, 10 (4), 563-572.

Hopley, A. B., \& Nicki, R. M. (2010). Predictive factors of excessive online poker playing. Cyberpsychology, Bebavior, and social networking, 13 (4), 379-385.

Jonsson, J. (2009, mars). Responsible gaming and gambling problems among 3000 Swedish Internet poker Players. Communication présentée au congrès de l'Alberta Gaming Research Institute, Banff conference on Internet Gambling, Alberta, Canada.

Joukhador, J., McCallum, F., \& Blaszczynski, A. (2003). Differences in cognitive distortions between problem gambling and social gamblers. Psychological Reports, 92, 1203-1214.

Kairouz, S., \& Nadeau, L. (2010). Enquête ENHJEU- Québec: portrait du jeu au Québec: prévalence, incidence et trajectoires sur quatre ans. Rapport de recherche remis au FQRSC.

Ladouceur, R., Sylvain, C., Boutin, C., \& Doucet, C. (2000). Le jeu excessif: comprendre et vaincre le gambling. Montréal, Québec: Les Éditions de l'homme.

Liley, J., \& Rakow, T. (2009). Probability estimation in poker: A qualified success for unaided judgment. Journal of behavioral decision making, 23, 496526.

Lloyd, J., Doll, H., Hawton, K., Dutton, W. H., Geddes, J. R., Goodwin, G. M., \& Rogers, R. D. (2010). Internet gamblers: A latent class analysis of their behaviours and health experiences. Journal of Gambling Studies, 26, 387-399.

Loto-Québec: EspaceJeux. (2011). Electronic References. Consulté le 20 février 2012, https://www.espacejeux.com/fr/on-joue-franc-jeu/.

Mattson, R. E., MacKillop, J., Castelda, B. A., Anderson, E. J., \& Donovick, P. J. (2008). The factor structure of gambling-related cognitions in an undergraduate university sample. Journal of Psychopathology and Behavioral Assessment, 30 (3), 229-234.

McCormack, A., \& Griffiths, M. D. (2012). What differentiates professional poker players from recreational poker players? A qualitative interview study. International Journal of Mental Health and Addiction, 10 (2), 243-257.

Michelat, G. (1975). Sur l'utilisation de l'entretien non directif en sociologie. Revue française de sociologie, 16, 229-247.

Mitrovic, D. V., \& Brown, J. (2009). Poker mania and problem gambling: A study of distorted cognitions, motivation and alexithymia. Journal of Gambling Studie, 25, 489-502.

Papineau, E. (2010). Enjeux de santé publique reliés à l'étatisation des jeux d'argent sur Internet. Québec. Consulté le 15 février 2012, http://www.inspq.qc.ca/ publications/notice.asp? $\mathrm{E}=\mathrm{p} \&$ NumPublication $=1055$.

Petry, N. (2005). Pathological gambling: Etiology, comorbidity, and treatment. Washington: American Psychological Association. 
Poupart, J. (1997). L'entretien de type qualitatif: considérations épistémologiques et méthodologiques. In J. Poupart, J.-P. Deslauriers, L.-H. Groulx, A. Laperrière, R. Mayer, \& A. P. Pires (eds), La recherche qualitative: enjeux épistémologiques et méthodologiques (173-209). Boucherville: Gaëtan Morin Editeur.

Radburn, B., \& Horsley, R. (2011). Gamblers, grinders, and mavericks: The use of membership categorisation to manage identity by professional poker players. Journal of Gambling Issues, 26, 30-50.

Responsible Gambling Council Poker Poll. (2006). One-in-five Ontarians Play Poker for Money. Onsulté le 8 décembre 2006, http://www.responsiblegambling.org/en/media/news_details.cfm? ID $=38 \&$ media $=1$.

Savoie-Zajc, L. (1998). L'échantillonnage. In Gauthier, B. (éd.), Recherche sociale, de la problématique à la collecte des données (3éd., 263-285). Québec: Presse de l'Université du Québec.

Shead, N. W., Hodgins, D. C., \& Scharf, D. (2008). Differences between poker players and non-poker-playing gamblers. International Gambling studies, 8 (2), 167-178.

Siler, K. (2010). Social and psychological challenges of poker. Journal of Gambling Studies, 26, 401-420.

Wiebe, J., Mun, P, et Kauffman, N. (2006). Gambling and problem gambling in Ontario 2005. Consulté le 8 décembre 2006, www.responsiblegambling.org/ en/research/rgcresearch.cfm.

Wood, R. T. A., \& Griffiths, M. D. (2008). Why Swedish people play online poker and factors that can increase or decrease trust in poker web sites: A qualitative investigation. Journal of Gambling Issues, 21, juillet, 80-99.

Wood, R. T. A., Griffiths, M. D., \& Parke, J. (2007). Acquisition, development, and maintenance of online poker playing in a student sample. Cyberpsycho$\log y$ and Behavior, 10 (3), 354-361.

ABSTRACT . Poker is currently the fastest growing gambling game in terms of popularity. Despite the large number of players, there is little scientific information available on poker players' gambling habits and their perceptions of this activity. In an effort to describe poker players' perceptions of their activity, qualitative interviews were conducted with 20 poker players. Analysis of the interview excerpts highlighted various themes related to players' perceptions of poker and other gambling games, the differences between the different types of poker, the role of poker in their lives and the qualities required to be a good poker player. Respondents' reports of their experiences led to three main findings: 1) poker differs from other gambling games in several aspects, 2) players do not seem concerned with the legal or illegal aspect of the activity and 3) poker players' views on their activity differ from those of their friends and families. Despite the limitations of this study, the participants' accounts clearly emphasize the specificities of the poker world.

KEYWORDS . Poker, gambling activity, qualitative, gambling, Internet. 
RESUMEN . Presentemente, el juego de azar y de dinero que está ganando mucha popularidad es el póquer. A pesar de sus numerosos adeptos, se dispone de pocas informaciones científicas sobre los hábitos de juego de los jugadores de póquer, asî como de la percepción que éstos tienen del juego. Con el fin de describir la percepción que los jugadores poseen de su actividad, se han realizado una serie de entrevistas cualitativas a una veintena de ellos. El análisis del discurso de éstos nos ha permitido resaltar diferentes temas en lo que respecta a su visión sobre otros juegos de azar, a su visión del póquer, las diferentes modalidades de póquer, a el rol del póquer en sus vidas y las cualidades necesarias para ser un buen jugador de póquer. A la luz de las experiencias relatadas por los entrevistados, surgen tres grandes constancias: 1) El póquer es un juego que se destaca del resto de los juegos en muchos aspectos 2) El aspecto legal o ilegal del juego no pareciera preocupar a los jugadores 3) La visión de los jugadores de su actividad difiere de la de sus allegados. A pesar de los límites de este estudio, el discurso de los participantes resalta bien las especificidades del mundo del póquer.

Palabras CLAVE . Póquer, juegos de azar y de dinero, cualitativo, gambling, Internet. 